\title{
基于未活化烯烃自由基加成/环化反应合成二氢 吲哚类化合物的研究进展
}

\author{
刘颖杰*,a 林立青 ${ }^{a}$ 韩莹徽 $^{a}$ 张 金金 $^{b}$ \\ $\left({ }^{a}\right.$ 哈尔滨商业大学药学院 哈尔滨 150076) \\ $\left({ }^{b}\right.$ 哈尔滨医科大学附属第一医院泌尿外科 哈尔滨 150001)
}

\begin{abstract}
摘要 二氢吲哚是一类具有广泛生物学特性的重要杂环，是许多天然产物和生物活性化合物的关键结构骨架. 有效的 二氢吲哚合成方法已成为广泛研究的主题. 描述了利用未活化烯烃合成各种官能化二氢吲哚的最新研究, 其涉及在氧 化剂存在下的自由基加成/环化反应，这些反应通常用容易获得的氧化剂和不同的金属或非金属作为催化剂，在中性条 件下进行反应.
\end{abstract}

关键词 二氢吲哚; 烯烃; 氧化剂; 官能团; 自由基

\section{Recent Advances of the Synthesis of Indolines by Unactivated Alkenes

\author{
Liu, Yingjie*,a $^{*}$ Lin, Liqing $^{a} \quad$ Han, Yinghui $^{a} \quad$ Zhang, Xin $^{b}$ \\ ( ${ }^{a}$ School of Pharmacy, Harbin University of Commerce, Harbin 150076) \\ ( ${ }^{b}$ Department of Urology, the First Affiliated Hospital of Harbin Medical University, Harbin 150001)
}

\begin{abstract}
Indolines, an important class of heterocycles with a wide range of biological properties, are a key structural motif in numerous natural products and biologically active compounds. As a result, efficient methods for indolines synthesis have been the subject of extensive studies. In this review, recent studies on the synthesis of various functionalized indolines using unactivated alkenes are described. It involves radical addition/cyclization reaction in the presence of oxidizing agent, which is usually carried out under neutral reaction conditions using readily available oxidizing agents and different transition metals or under metal-free as catalysts.

Keywords indolines; alkenes; oxidizing agent; functional group; free radical
\end{abstract}

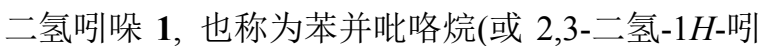
哚或 1-氮杂狮二酮), 是具有双环结构的芳香杂环骨架, 双环结构由五元含氮杂环稠合的六元苯环组成, $\mathrm{p} K_{\mathrm{a}}$ 值 为 $4.9(\text { 图 } 1)^{[1]}$.

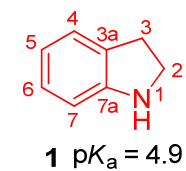

图 1 二氢吲哚结构图

Figure 1 Indoline nucleus

吲哚类化合物广泛存在于自然界中, 很多天然产物
和药物分子都含有二氢吲哚的结构骨架(图 2), 二氢吲 哚的衍生物具有较好的药理活性和生理活性, 例如在抗 菌、抗惊厥、抗肿瘤和抗炎等方面具有较好的活性等 ${ }^{[2,3]}$. 具有二氢吲哚骨架的化合物在医药、农药、染料等方面 具有良好活性 ${ }^{[4]}$. 在吲哚化学中, 制备二氢吲哚骨架比 合成其他衍生物如吲哚酮更具挑战性 ${ }^{[57]}$. 获得二氢吲 哚骨架的主要方法: (1)吲哚的脱芳构化. 然而使用非环 状化合物制备二氢吲哚类衍生物的实例很少 ${ }^{[8] ;}$; (2)利用 吲哚酮还原二氢吲哚. 此方法需要严苛的条件 ${ }^{[9]}$. 因此 对二氢吲哚及其衍生物的合成研究仍具备重要意义.

烯烃的双官能化是合成具有生物活性的杂环非常

* Corresponding author. E-mail: liuyj691@nenu.edu.cn

Received April 6, 2019; revised May 27, 2019; publishend online June 12, 2019

Project supported by the Program of Young Innovators of Education Department of Heilongjiang Province (No. UNPYSCT-2016181).

黑龙江省创新人才(No. UNPYSCT-2016181)资助项目. 


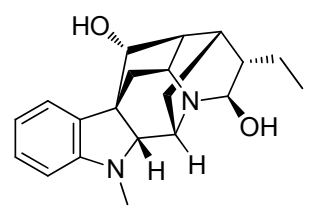

2 (+)-Ajmaline

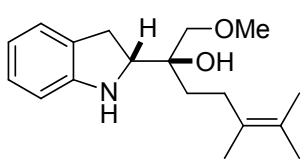

4 (+)-Benzastatin

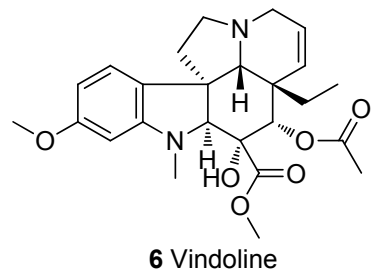

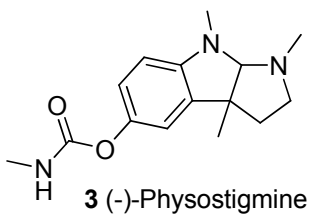

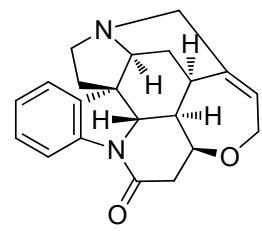

5 Strychnine

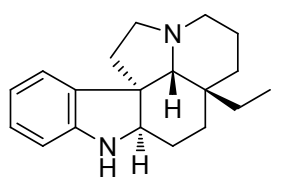

7 (+)-Aspidospermidine

图 2 具有二氢吲哚结构的代表性天然生物碱

Figure 2 Representative natural alkaloids with the indoline framework

重要的方法 ${ }^{[10]}$. 特别是, 活性烯烃的邻位双官能化反应 是过去几十年中获得分子复杂性的有力手段, 而在未活 化的烯烃中同时引入两种官能团的意义同样重要 ${ }^{[11]}$. 最 近, 多个课题组研究使用未活化的烯烃作为自由基受 体，使用铁或银等金属催化剂或在无金属条件下，通过 自由基加成/环化反应，使未活化的烯烃双官能化来构 建二氢吲哚. 此方法可以将多种官能团如三氟甲基、多 氯甲基、氧基、羰基和磷酰基等引入到二氢吲哚骨架中, 来构建具有各种官能团的二氢吲哚类衍生物. 根据烯烃 双官能化转化类型, 主要分为两种: (a)在碳-碳双键上 加入碳自由基, 然后进行芳基化, 通过烯烃 1,2-碳碳官 能化合成二氢吲哚; (b)在碳一碳双键上加入杂原子和碳 自由基, 然后进行芳基化, 通过烯烃的 1,2-碳杂官能化 合成二氢吲哚(Scheme 1). 本综述按照上述烯烃的双官 能化的转化类型, 结合各种自由基、反应机理介绍不同 的 C(3)取代的二氢吲哚分子骨架的构建, 对近年来未活 化烯烃通过自由基加成/环化反应合成二氢吲哚的研究 进展进行综述.

\section{2 通过烯烃的 1,2-碳碳官能化合成二氢吲哚}

\section{1 未活化烯烃的 1,2-三氟甲基化/芳基化构建 $C$ (3)三 氟甲基/烷基双取代二氢吲哚}

三氟甲基已被认为是可以增强母体分子的代谢稳 定性、亲脂性和生物利用度的重要药效团 ${ }^{[12]}$. 虽然研究 开发将三氟甲基结合到生物活性分子中的方法受到广 泛关注, 然而成功的例子却很少见. 活化烯烃的三氟甲 基化邻位双官能化已被证明是获得分子复杂性的有力 策略 ${ }^{[13 \sim 16]}$, 而 3-(2,2,2-三氟乙基)二氢吲哚的合成仍然

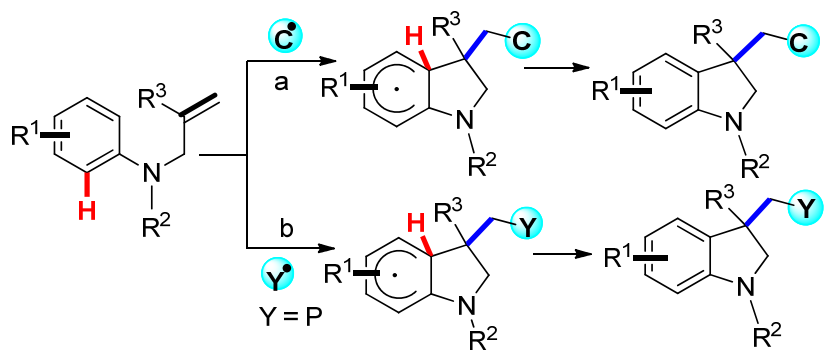

图式 1 未活化的烯烃通过自由基加成/环化反应双官能化 Scheme 1 Difunctionalization of unactivated alkenes through radical addition/cyclization

是一项重大挑战，可能因为未活化的烯烃易于聚合和双 键转移.

2001 年, Zard 及其同事 ${ }^{\left[{ }^{[7]}\right.}$ 报道了在过氧化月桂酰作 为引发剂存在下, 在回流的 $1,2-$ 二氯乙烷(DCE)中加热 黄原酸酯和受保护的 $N$-烯丙基对氟苯胺, 得到中间产 物 8 , 产率为 $64 \%$. 将 $\mathbf{8}$ 暴露在过氧化月桂酰中, 最终得 到二氢吲哚的产率为 $60 \%$ (Scheme 2).

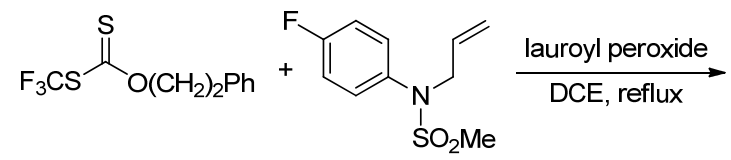

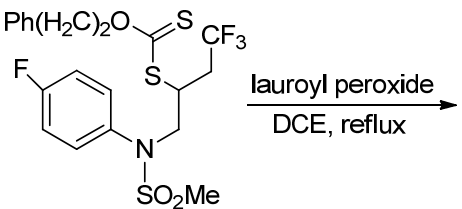

$8,64 \%$

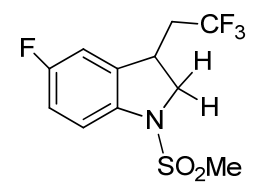

$60 \%$, only 1 example
图式 2 黄原酸酯和 $N$-烯丙基对氟苯胺的多氟烷基化 Scheme 2 Polyfluoroalkylation of xanthate and $N$-allyl $p$-fluoroaniline

2013 年, Sodeoka 课题组 ${ }^{[18]}$ 报道了使用 $\mathrm{CuI} / \mathrm{Togni}$ 试剂催化的未活化烯烃的三氟甲基化，以及独特的 1,6氧三氟甲基化反应. 通过烯烃和芳基之间的轨道相互作 用加速反应，有利于三氟甲基化反应以及分子内 $\mathrm{C}-\mathrm{C}$ 键形成. 该反应体系被应用于 $N$-Boc-烯丙基苯胺和 TBS 保护的烯丙基苯胺, 得到对应的三氟甲基化二氢吲哚, 产率分别为 $85 \%$ 和 $87 \%$ (Scheme 3). 2017 年，该课题组 [19]报道了使用全氟酸酐(TFAA)作为全氟烷基源，用于 $N$-芳基烯丙胺的三氟烷基化，得到三氟烷基化的二氢吲 哚(Scheme 4).

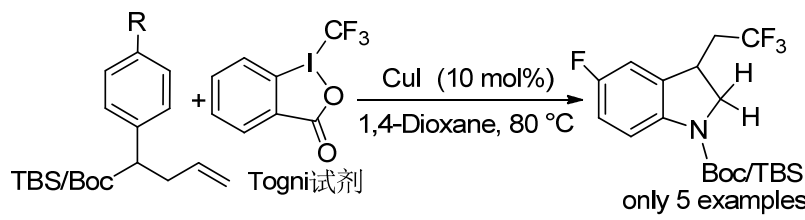

图式 3 铜催化的未活化烯烃的三氟甲基化

Scheme 3 Copper-catalyzed trifluoromethylation of unactivated alkenes 


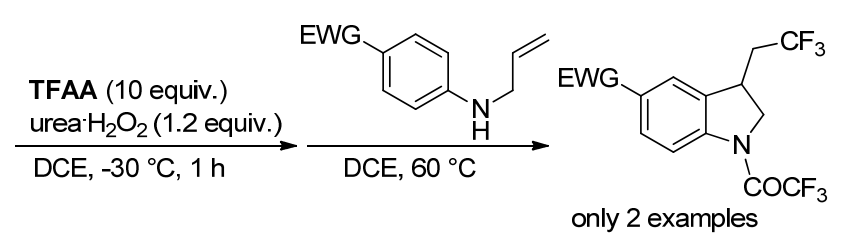

图式 4 TFAA 与 $N$-芳基烯丙胺的三氟烷基化 Scheme 4 Trifluoroalkylation of TFAA with $N$-arylallylamine

2013 年, $\mathrm{Fu}$ 及其同事 ${ }^{[20]}$ 研究报道了以铜粉作为催 化剂, 使用 Umemoto 试剂作为 $\mathrm{CF}_{3}$ 源, 使用亚硝酸异戊 酯( $i$-AmONO)进行原位重氮化反应, 得到三氟甲基化的 二氢吲哚, 产率为 $62 \%$ (Scheme 5). 此反应条件苛刻, 需使用大量的的铜才能顺利完成反应, 而且底物的范围 比较窄, 成本较高.

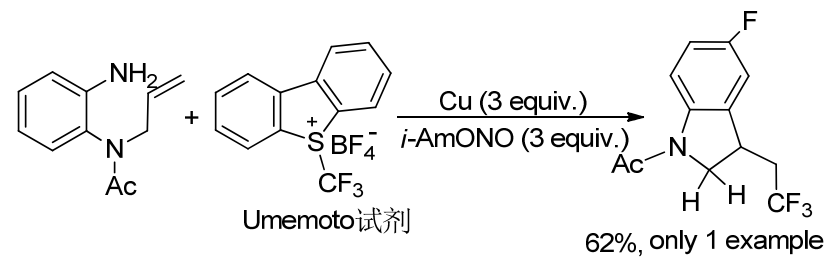

图式 5 Umemoto 和未活化烯烃的多氟烷基化反应 Scheme 5 Polyfluoroalkylation of Umemoto and unactivated alkenes

2017 年, Cheng 课题组 ${ }^{[21]}$ 描述了有效且高度实用的 钯催化未活化的烯烃的芳基全氟烷基化, 得到三氟甲基 化二氢吲哚的产率为 $85 \%$ (Scheme 6). 该反应温和地进 行且没有芳基的电子活化, 具有高通用性, 低成本的氟 烷基化来源和良好的官能团相容性. 但该反应涉及到有 毒气体以及苛刻的反应条件.
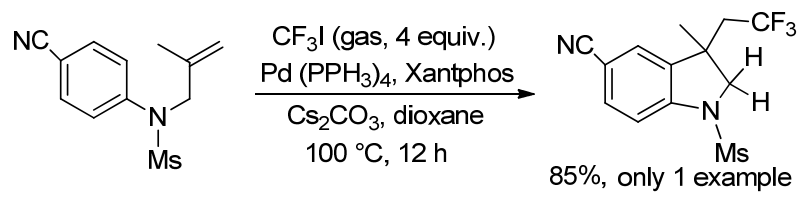

图式 6 钯催化的未活化的烯烃的芳基全氟烷基化

Scheme 6 Palladium-catalyzed aryl perfluoroalkylation of unactivated alkenes

尽管利用未活化烯烃为起始物成功地构建了二氢 吲哚类化合物, 但是往往反应程序繁琐, 底物范围窄, 成本高, 或反应条件苛刻、毒性等条件. 这样不仅导致 在底物的兼容性方面有诸多限制, 而且也降低了这类方 法的实用性.

2018 年, Liang 及其同事 ${ }^{[22]}$ 报道了未活化的烯烃的 无金属串联三氟甲基化/芳基化, 以 $\mathrm{CF}_{3} \mathrm{SO}_{2} \mathrm{Na}$ 作为三氟 甲基化剂和 $N$-烯丙基苯胺的未活化烯烃作为自由基受 体, 发生三氟甲基烷基化环化级联反应, 反应以高收率 获得含 $\mathrm{CF}_{3} \mathrm{CH}_{2}$ 的二氢吲哚和 1,2,3,4-四氢异喹啉 (Scheme 7). 使用廉价且稳定的固体 $\mathrm{CF}_{3} \mathrm{SO}_{2} \mathrm{Na}$ (Lang- lois'试剂)作为三氟甲基化剂. 该反应具有条件温和，操 作简便，成本低和底物范围广的特点.
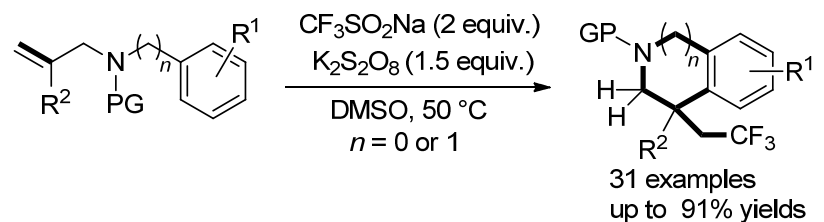

(a) Radical trapping experiments

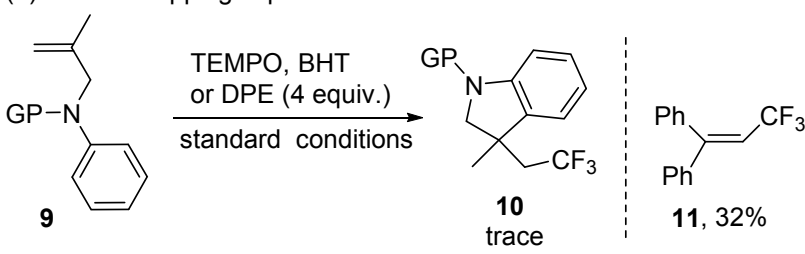

(b) Proposed mechanism

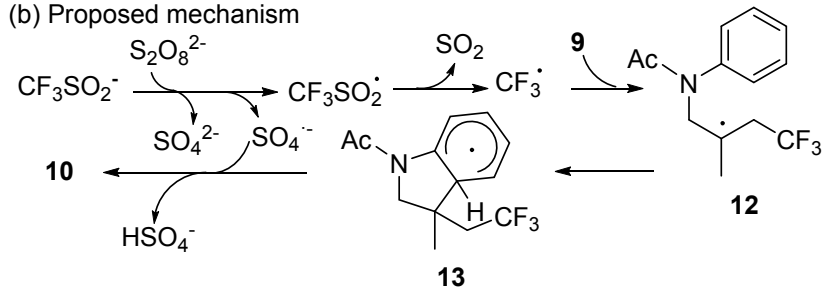

图式 7 未活化烯烃的分子内三氟甲基化/芳基化串联反应 Scheme 7 Intramolecular trifluoromethylation/arylation cascade reaction of unactiveted alkenes

自由基捕获实验表明, 加入 4 equiv.的 2,2,6,6-四甲 基哌啶-1-氧基(TEMPO)和 2,6-二叔丁基-4-甲基苯酚 (BHT), 完全抑制标准条件下的模型反应. 在 DPE 实验 中, 1,1 -二苯基乙烯(DPE)作为自由基清除剂, $\mathrm{DPE}^{-\mathrm{CF}_{3}}$ 加合物 11 以 $32 \%$ 的产率分离. 根据上述结果和文献报 告 ${ }^{[15,23]}$, 提出了一种可能机制. 最初, $\mathrm{CF}_{3} \mathrm{SO}_{2} \mathrm{Na}$ 与 $\mathrm{K}_{2} \mathrm{~S}_{2} \mathrm{O}_{8}$ 反应释放出硫酸盐二价阴离子, 硫酸根阴离子和 三氟甲磺酰自由基反应，释放出一个二氧化硫分子得到 三氟甲基自由基，与 $N$-烯丙基苯胺 9 的未活化双键反应 得到烷基 12.12 通过分子内自由基进行环化产生脱芳基 化的芳基 13. 随后 13 脱氢转移到硫酸根阴离子释放出 二氢吲哚 10 以及硫酸氢根阴离子.

\section{2 未活化烯烃的 1,2-烷基/芳基化构建 C(3)烷基双取 代二氢吲哚}

腈类物质在材料、药物和有机合成方面的应用日益 增多，并且已经致力于制备含氧基的吲哚衍生物. 2012 年, $\mathrm{Liu}$ 及其同事 ${ }^{[24]}$ 使用 $\mathrm{Pd}(\mathrm{OAc})_{2} / \mathrm{PhI}\left(\mathrm{O}_{2} \mathrm{Ct} \text { - } \mathrm{Bu}\right)_{2} / \mathrm{AgF}$ 系 统将未活化的 $N$-烯丙基苯胺烯烃进行氰基烷基化/环化 反应, 以乙腈作为氧化物源生成氧化二氢吲哚(Scheme 8). 腈溶剂是理想的氰基烷基化试剂, 但为了激活它们 的 $\mathrm{C}-\mathrm{H}$ 键, 使用了贵金属钯催化剂和超化学计量的 $\mathrm{AgF}$ ，以及高价碘试剂．此外，这种极性反应对腈分子 中的空间位阻敏感, 并且不能将二级或三级腈部分引入 二氢吲哚骨架. 


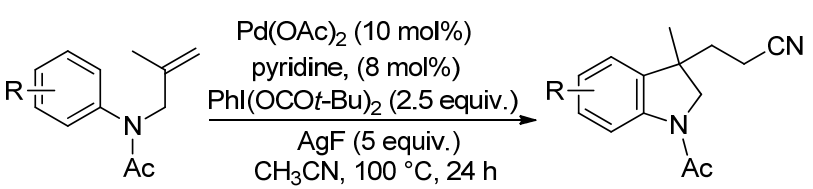

图式 8 钯催化的未活化烯烃的氧化芳基烷基化 Scheme 8 Palladium-catalyzed oxidative arylalkylation of unactivated alkenes

偶氮二异丁腈(AIBN) 是聚合物化学 ${ }^{[25]}$ 和自由基有 机合成 ${ }^{[26]}$ 中最广泛使用的自由基引发剂之一. 传统上, 它仅引发自由基过程, 不参与反应, 因为源自 AIBN 的 异丁腈基团通常被认为是无活性的，部分原因是空间位 阻 ${ }^{[27]}$. 然而, 过去几年, AIBN 作为三级腈源可以构建一 个 $\alpha$-氰基季碳中心 ${ }^{[28]}$. $\mathrm{Li}$ 课题组 ${ }^{[29]}$ 将 $N$-烯丙基苯胺的未 活化烯烃发生氰基烷基化/环化级联, 生成 3-氰基烷基 二氢吲哚(Scheme 9).

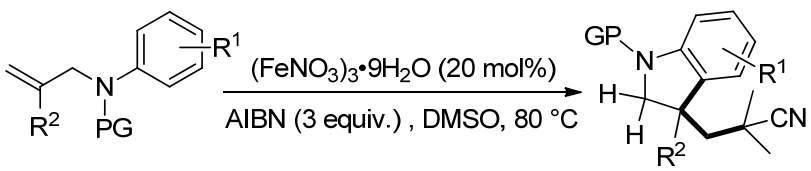

(a) Radical trapping experiments

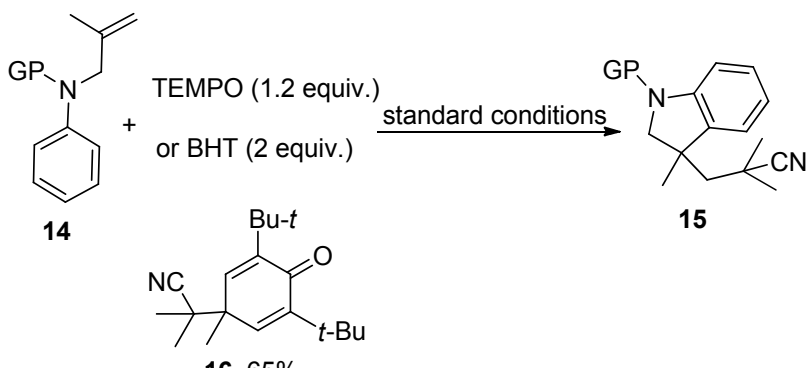

(b) Proposed mechanism

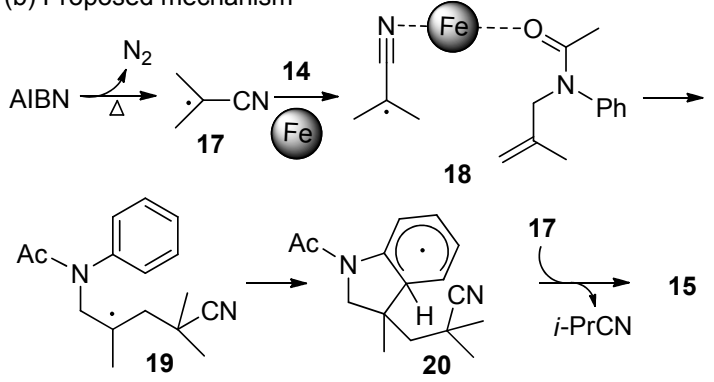

图式 9 未活化烯烃和偶氮二异丁腈的自由基氰甲基化/芳基 化反应

Scheme 9 Radical cyanomethylation/arylation reaction of unactivated alkenes with azobisisobutyronitrile

通过对照实验以证实该环化的自由基性质. 以 1.2 equiv. 的 2,2,6,6-四甲基哌啶-1-氧基(TEMPO)或 2 equiv. 的 2,6-二叔丁基-4-甲基苯酚(BHT)作为自由基抑制剂, 几乎完全抑制了模型反应. 此外, 氰基异丙基-BHT 加 合物 16 以 $65 \%$ 的产率分离, 作为 BHT 实验中的唯一产 物.
根据上述结果和以前的报告 ${ }^{[28]}$, 提出了一种可能 的机制. 最初, AIBN 的热分解释放出一个氮分子，得到 异丁腈自由基 17. 17 进攻 $N$-烯丙基苯胺 14 的双键，与 18 和铁络合形成中间产物 19, 随后 19 闭环得到中间体 20.20 的氢原子转移到 17 , 释放出二氢吲哚产物 15 和异 丁腈.

\section{3 未活化烯烃的 1,2-多氯甲基化/芳基化构建 C(3)烷 基双取代二氢吲哚}

含卤素的化合物在天然产物和合成的有机化合物 中广泛存在, 并表现出多种生物活性 ${ }^{[30]}$. 其中, 具有二 氯或三氯甲基的多卤化合物存在于许多具有生物活性 的天然产物, 、药物以及农药中 ${ }^{[31]}$. Pan 课题组 ${ }^{[32]}$ 报道了 使用二氯甲烷、氯仿和四氯甲烷作为多氯甲基源，在无 金属和无添加剂条件下，利用双官能化未活化的烯烃来 合成二氯和三氯甲基化二氢吲哚(Scheme 10).

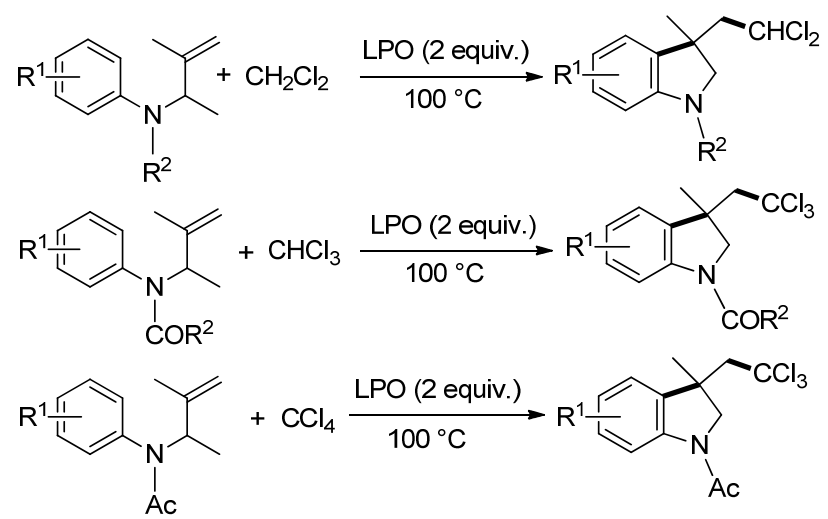

图式 10 无金属条件下未活化烯烃的自由基级联氯甲基化反 应

Scheme 10 Metal-free radical cascade chloromethylation of unactivated alkenes

通过对照实验来研究反应机理(Scheme 11). 分子内 和分子间竞争动力学同位素效应(KIE) 实验表明. $\mathrm{CH}_{2} \mathrm{Cl}_{2}$ 与 $\mathrm{CD}_{2} \mathrm{Cl}_{2}$ 的分子间 $\mathrm{KIE}\left(k_{\mathrm{H}} / k_{\mathrm{D}}=6.7\right)$ 差异显著, 在 $\mathrm{CH}_{2} \mathrm{Cl}_{2}$ 和 $\mathrm{CD}_{2} \mathrm{Cl}_{2}$ 的单独反应中 $\mathrm{KIE} k_{\mathrm{H}} / k_{\mathrm{D}}=5.2$. 结果 表明，该反应的速率决定步骤是 DCM 中 $\mathrm{sp}^{3}-\mathrm{C}-\mathrm{H}$ 键的 裂解，而不是芳基中 $\mathrm{sp}^{2}-\mathrm{C}-\mathrm{H}$ 键的裂解 ${ }^{[33]}$.

基于以上结果，提出了反应机理. 首先 LPO 均裂解 产生月桂酰基, 然后, 月桂酰基从 $\mathrm{CH}_{2} \mathrm{Cl}_{2}$ 中夺去氢原子 产生二氯甲基自由基 23, 23 进攻 $N$-烯丙基苯胺 21 的双 键得到自由基中间体 24, 24 分子内环化产生自由基中间 体 25. 最后, 夺氢产生二氯甲基化的二氢吲哚产物 22 .

\section{4 未活化烯烃的 1,2-羰基化/芳基化构建 C(3)羰基/ 烷基双取代二氢吲哚}

羰基 $\mathrm{C}\left(\mathrm{sp}^{2}\right)-\mathrm{H}$ 键是高度反应性的化学键, 广泛用 于合成 ${ }^{[34]}$, 含羰基的二氢吲哚是药物和天然产物中的 
(a) Preliminary mechanism studies

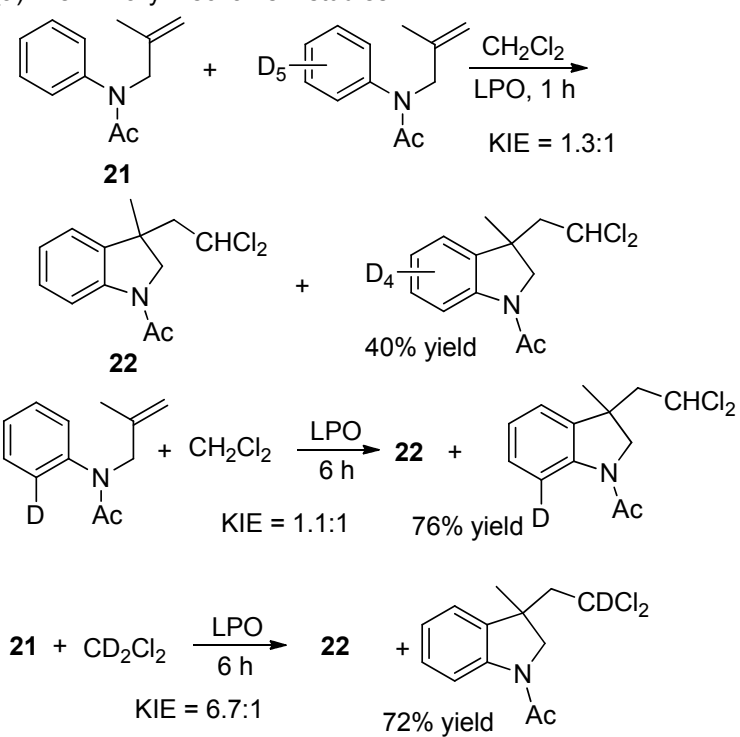

(b) Proposed mechanism

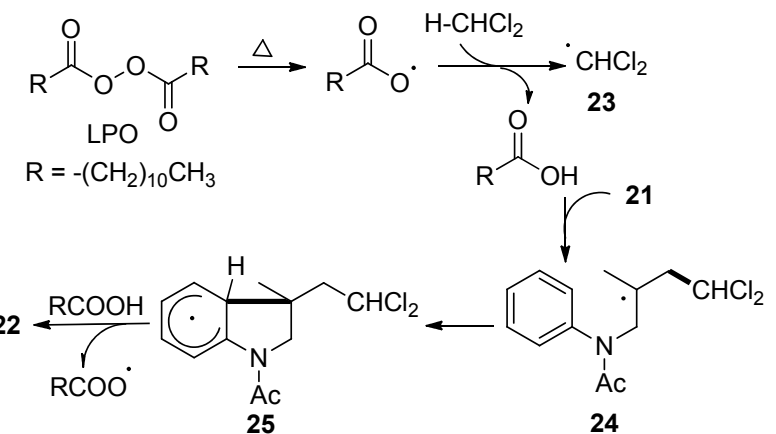

图式 11 未活化烯烃的自由基级联/氯甲基化反应的可能机制 Schme 11 Possible mechanism for radical cascade chloromethylation of unactivated alkenes

常见结构骨架，也是有机合成中的通用中间体.

2018 年, Pan 课题组 ${ }^{[35]}$ 研究报道了不含金属和酸的 $N$-烯丙基苯胺与丙酮的氧化加成/环化, 产生一系列 3-(3-氧代丁基)官能化的二氢吲哚. 该反应的特征在于 容易产生与未活化的双键反应的酮基, 得到 3-(3-氧代 丁基)二氢(吲哚, 是天然产物和药物的重要结构骨架 (Scheme 12).

通过对照实验以研究反应机理, 添加自由基清除剂 TEMPO 成功地抑制了模型反应. 分子间动力学同位素 实验表明, 芳烃 $\mathrm{C}-\mathrm{H}$ 键的 $k_{\mathrm{H}} / k_{\mathrm{D}}$ 为 1.3 和 1.1 , 说明自由 基中间体参与反应, 并且芳烃 $\mathrm{C}-\mathrm{H}$ 键的裂解不参与速 率确定步骤. 另一方面, 在该过程中观察到丙酮的大的 动力学同位素效应, 表明丙酮中 $\mathrm{sp}^{3}-\mathrm{C}-\mathrm{H}$ 键的裂解参 与该转化的速率确定步骤.

可能的机理如 Scheme12 所示. 首先, 29均裂产生的 月桂酰自由基, 与丙酮 $\mathbf{2 7}$ 的 $\alpha-\mathrm{H}$ 形成丙酮自由基 $\mathbf{2 9}$. 然 后, 29 与 $N$-烯丙基苯胺 26 发生分子间加成产生自由基 中间体 30. 分子内自由基环化得到中间体 31, 其经历氧 化脱氢得到产物 28.<smiles>[R1]c1cccc(N([CH])C(=C)C)c1</smiles>
up to $85 \%$ yields

(a) Preliminary mechanism studies

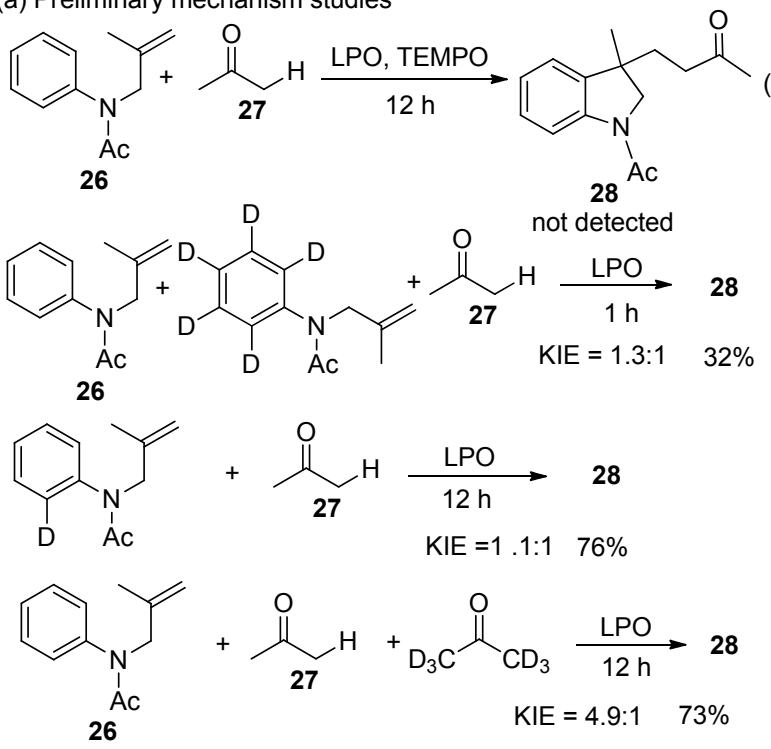

(b) Proposed mechanism

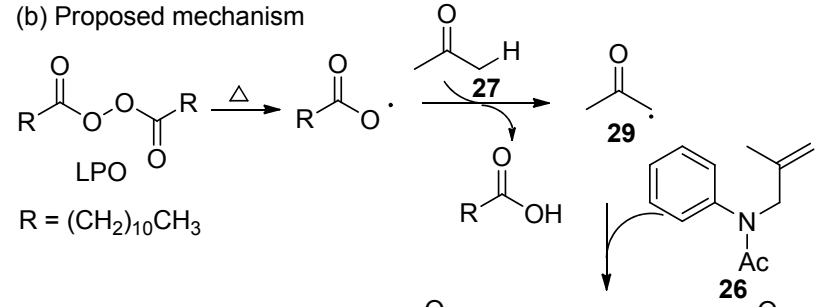

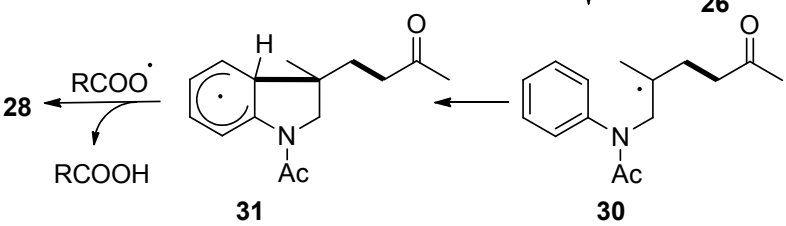

图式 12 无金属条件下未活化烯烃与丙酮的氧化自由基级联 加成反应

Scheme 12 Metal-free oxidative radical cascade addition reaction of unactivated alkenes with acetone

\section{3 未活化烯烃的碳杂官能化合成二氢吲哚}

\section{1 未活化烯烃的 1,2-膦酰化/芳基化构建 C(3)膦酰 基/烷基双取代二氢吲哚}

含磷有机化合物广泛存在于药物和农用化学品中; 吲哚基和磷基的结合产生了一系列具有抗癌活性 ${ }^{[36]}$ 、 PET 显像 ${ }^{[37]}$ 、抗艾滋病毒 ${ }^{[38]}$ 、抗病毒 ${ }^{[39]}$ 、抗分枝杆菌 ${ }^{[40]}$ 等化合物 ${ }^{[41]}$ 和线粒体靶向制剂 ${ }^{[42]}$ 等. 这些益处推动了 生成含磷吲哚啉的新方法的发展.

自由基亲核取代或 $\mathrm{S}_{\mathrm{RN}} 1$ 反应是具有自由基和自由 基阴离子作为中间体的链过程, 该链式过程需要引发 剂. 最常用的引发方法是液氨中的碱金属化学引发, 阴 
极的电化学引发和光引发. Rossi 研究小组 ${ }^{[43]}$ 于 2002 年 报道了使用 $\mathrm{Ph}_{2} \mathrm{P}^{-}$作为亲核试剂, 通过自由基亲核取代 机理，由邻位官能化的卤代芳基化合物成功制备 3-位被 磷酰基取代的二氢吲哚(Scheme 13).

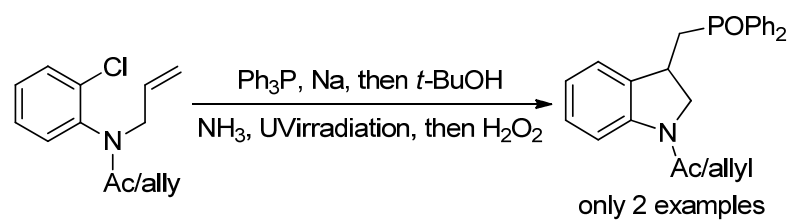

图式 13 串联环化- $\mathrm{S}_{\mathrm{RN} 1}$ 反应合成二氢吲哚

Scheme 13 Syntheses of indolines by tandem ring closure- $\mathrm{S}_{\mathrm{RN} 1}$ reactions

2003 年, Zard 及其同事 ${ }^{[44]}$ 报道了黄原酸的自由基交 换机理. 用少量过氧化物作为引发剂, 黄原酸酯与未活 化烯烃直接合成官能化的偕二膦酸酯. 在回流条件下于 1,2-二氯乙烷中加入偕二膦酸酯与过氧化物, 在过氧化 物的诱导作用下发生自由基加成/环化反应, 得到相应 的二氢吲哚, 产率 $62 \%$ (Scheme 14).

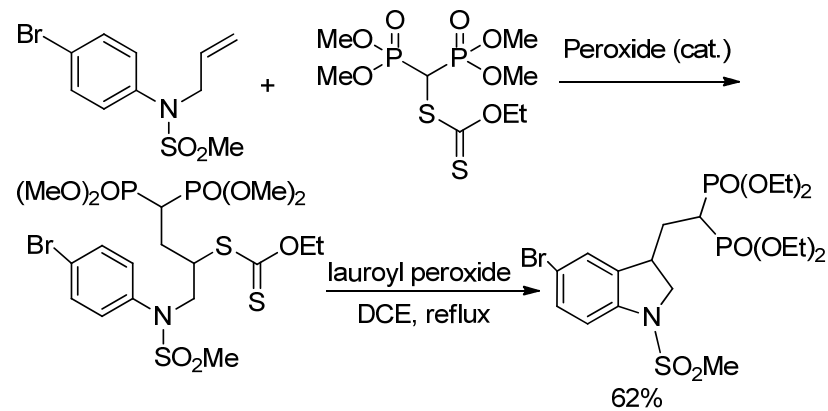

图式 14 黄原酸酯与未活化烯烃合成磷酰基化二氢吲哚

Scheme 14 Synthesis of phosphorylated indolin by xanthates and unactivated alkenes

2010 年, Curran 小组 ${ }^{[45]}$ 报道了邻碘苯胺在自由基条 件下与 $\mathrm{Me}_{3} \mathrm{SnPPh}_{2}$ 反应，经历芳基自由基磷酰化、氧化 得到磷酰基化二氢吲哚衍生物, 收率为 70\%. 2011 年, 该课题组 ${ }^{[46]}$ 用 $\mathrm{Me}_{3} \mathrm{SnPPh}_{2}$ 和引发剂 V-40 在苯中处理二 碘酰苯胺. 为了简化分析和产物分离, 将反应混合物用 $\mathrm{S}_{8}$ 处理, 得到了双硫化膦二氢吲哚衍生物(Scheme 15).

随着对未活化烯烃双官能化的深入研究, Liang 课 题组 ${ }^{[47]}$ 开发了一种银催化的 $N$-烯丙基苯胺的磷酸化自 由基加成/环化反应，直接获得 3-膦酰基烷基二氢吲哚, 未活化的烯烃用作自由基受体, H-膦酸盐或 H-膦氧化物 用作自由基前体. 该方案操作简单、底物范围广、外部 选择性高、很容易进行大规模合成(Scheme 16).

通过一些对照实验以探测反应机理. 在标准条件 下, 通过加入 TEMPO 或 BHT 作为自由基清除剂, 模型 反应被完全抑制. 基于上述观察和先前的报告 ${ }^{[48]}$, 提出<smiles>Cc1cc(C)c(N(C2C=CCCC2)C(C)(C)C)c(I)c1</smiles>

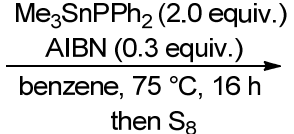

$\mathrm{Ph}_{2} \mathrm{SP}$<smiles>Cc1cc(I)c(N(C)C2C=CCCC2)c(I)c1</smiles><smiles>Cc1cc(C)c2c(c1)C1C(C)CCCC1N2C(C)(C)C</smiles>

$\mathrm{Ph}_{2} \mathrm{SP}$

图式 $15 \mathrm{Me}_{3} \mathrm{SnPPh}_{2}$ 进行芳基自由基磷酰化氧化得到二氢吲 哚

Scheme 15 Indolin was obtained by aryl radical phosphorylation oxidation of $\mathrm{Me}_{3} \mathrm{SnPPh}_{2}$

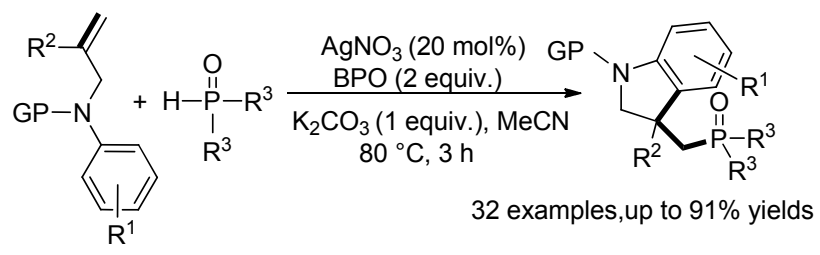

(a) Radical trapping experiments
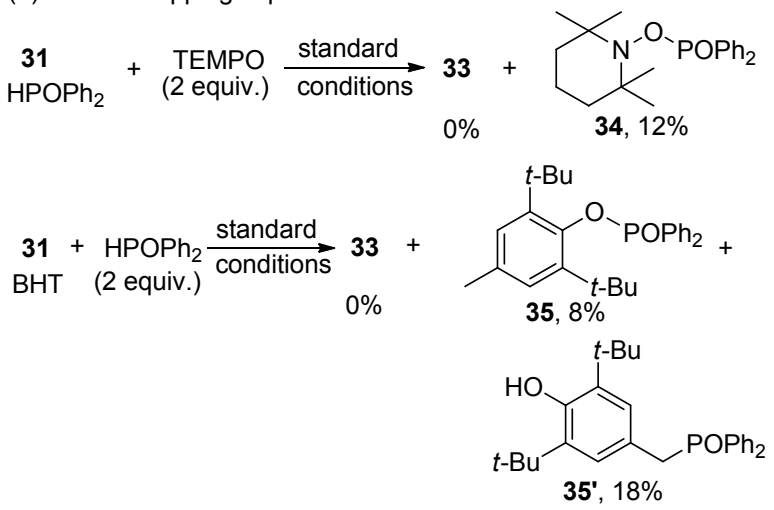

(b) Proposed mechanism

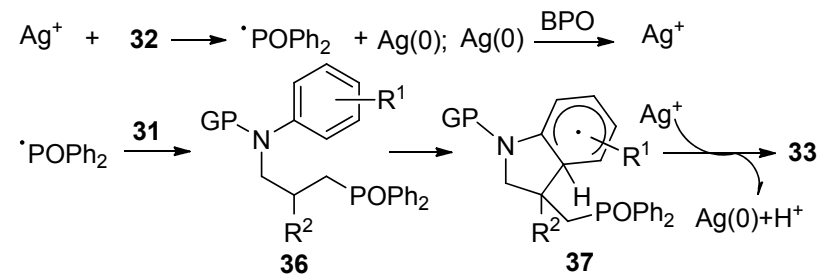

图式 16 银催化的未活化烯烃的自由基芳基磷酸化 Scheme 16 Silver-Catalyzed radical arylphosphorylation of unactivated alkenes

了可能的机制. 开始, 膦酸盐或膦氧化物 32 与 $\mathrm{AgNO}_{3}$ 反应得到磷酰自由基，磷酰自由基与 $N$-烯丙基苯胺 31 双键反应，形成具有 $\mathrm{C}-\mathrm{P}$ 键的自由基中间体 36, 36 的 苯环通过分子内自由基进行环化得到闭环中间体 $\mathbf{3 7}, \mathbf{3 7}$ 与 $\mathrm{Ag}^{+}$发生单电子转移(SET) 释放出二氢吲哚产物 33、 质子和 $\mathrm{Ag}(0)$. 最后通过 $\mathrm{BPO}$ 将 $\mathrm{Ag}(0)$ 氧化成 $\mathrm{Ag}^{+}$以完 成催化性循环. 


\section{4 结论与展望}

在过去的几年中, 使用未活化的烯烃作为自由基受 体, 通过自由基加成/环化反应合成二氢吲哚及其衍生 物的反应策略取得了很大进展。这些新方法由碳或杂原 子基团引发, 容易将许多官能团(包括烷基、羰基、三氟 甲基、二氯甲基、氭基和磷酰基)引入到二氢吲哚骨架 中. 鉴于反应底物 $N$-芳基丙烯酰胺具有廉价、易于制备 和储存等特点, 该类型的反应必将在医药中间体、功能 性有机材料等的合成中得到更加广泛的应用.

尽管在使用自由基加成/环化反应合成二氢吲哚方 面取得了重大进展, 但在未活化烯烃的双官能化转化过 程中，对非绿色氧化剂的依赖性非常突出(如过氧化物、 过硫酸钾、高价碘或叔丁基). 因此, 开发涉及使用绿色 氧化剂(例如 $\mathrm{O}_{2}$ 过氧化氢或其他可持续氧化剂)的新的 有效自由基加成/环化反应策略变得尤为重要. 该领域 的重要机遇和挑战还将涉及开发更加绿色有效的催化 剂和发现更多新的功能基团, 以便进一步研究未开发的 自由基加成/环化反应.

\section{References}

[1] Silva, T. S.; Rodrigues, M. T.; Santos, H.; Zeoly, L. A.; Almeida, W. P.; Barcelos, R. C.; Gomes, R. C.; Fernandes, F. S.; Coelho, F. Tetrahedron 2019, 75, 2553.

[2] Collins, M. A.; Hudak, V.; Bender, R.; Fensome, A.; Zhang, P.; Miller, L. Med. Chem. Lett. 2004, 14, 2185.

[3] Tokunaga, T.; Hume, W. E.; Umezone, T.; Okazaki, K.; Ueki, Y.; Kumagai, K. J. Med. Chem. 2001, 44, 4641.

[4] (a) Wang, T.; Xu, Q.; Yu, P. Org. Lett. 2001, 3, 345.

(b) Bui, T.; Syed, S.; Barbas, C F. J. Am. Chem. Soc. 2009, 131, 8758.

(c) Toda, N.; Ori, M.; Takami, K.; Tago, K.; Kogen, H. Org. Lett. 2003, 5, 269.

(d) Zhang, H.; Boonsombat, J.; Padwa, A. Org. Lett. 2007, 9, 279.

(e) Langlois, N.; Gueritte, F.; Langlois, Y. J. Am. Chem. Soc. 1976, 98, 7017 .

(f) Marino, J. P.; Rubio, M. B.; Cao, G. J. Am. Chem. Soc. 2002, 124,13398

[5] (a) Van Order, R. B.; Lindwall, H. G. Chem. Rev. 1942, 30, 69.

(b) Taber, D. F.; Tirunahari, P. K. Tetrahedron 2011, 67, 7195.

(c) Petrini, M. Chem. Eur. J. 2017, 23, 16115.

(d) Leitch, J. A.; Bhonoah, Y.; Frost, C. G. ACS Catal. 2017, 7, 5618 .

[6] (a) Kirillova, M. S.; Miloserdov, F. M.; Echavarren, A. M. Org. Chem. Front. 2018, 5, 273.

(b) Song, J.; Chen, D.; Gong, L. Natl. Sci. Rev. 2017, 4, 381.

(c) Corsello, M. A.; Kim, J.; Garg, N. Chem. Sci. 2017, 8, 5836.

(d) Homer, J. A.; Sperry, J. J. Nat. Prod. 2017, 80, 2178.

[7] (a) Singh, A. K.; Raj, V.; Saha, S. Eur. J. Med. Chem. 2017, 142, 244.

(b) Sravanthi, T. V.; Manju, S. L. Eur. J. Pharm. Sci. 2016, 91, 1.

(c) Sugimoto, S.; Naganuma, M.; Kanai, T. J. Gastroenterol. 2016, 51,853 .

(d) Megna, B. W.; Carney, P. R.; Nukaya, M.; Geiger, P.; Kennedy, G. D. J. Surg. Res. 2016, 204, 47.

[8] (a) Zheng, C.; Zheng, S. Y. Chem 2016, 1, 830.

(b) Liang, X.; Zheng, C. ; You, S. Chem.-Eur. J. 2016, 22, 11918.

(c) Roche, S. P.; Tendoung, J. Y.; Treguier, B. Tetrahedron 2015, 71, 3549 . (d) Ding, Q. ; Zhou, X. ; Fan, R. Org. Biomol. Chem. 2014, 12, 4807.

[9] (a) Wang, L.; Li, S.; Blmel, M.; Puttreddy ,R.; Peuronen, A.; Rissanen,K.; Enders, D. Angew. Chem., Int. Ed. 2017, 56, 8516.

(b) Zhao, B.; Du, D.; Chem. Commun. 2016, 52, 6162.

(c) Kayal, S.; Mukherjee, S.Org. Biomol. Chem. 2016, 14, 10175. (d) Du, D.; Jiang, Y.; Xu, Q.; Tang, X.; Shi, M. ChemCatChem 2015, 7, 1366.

[10] For selected reviews on difunctionalization of alkenes, see: (a) Kolb, H. C.; Van Nieuwenhze, M. S.; Sharpless, K. B. Chem. Rev. 1994, 94, 2483.

(b) Beccalli, E. M.; Broggini, G.; Martinelli, M.; Sottocornola, S. Chem. Rev. 2007, 107, 5318.

(c) Jacques, B.; Muniz, K. In Catalyzed Carbon-Heteroatom Bond Formation, Ed.: Yudin, A. K., Wiley-VCH, Weinheim, 2010, 119. (d) McDonald, R. I.; Liu, G.; Stahl, S. S. Chem. Rev. 2011, 111, 2981.

(e) Chen, J.-R.; Yu, X.-Y.; Xiao, W.-J. Synthesis 2015, 47, 604.

(f) Sun, K.; Luan, B.; Liu, Z.; Zhu, J.; Du, J.; Bai, E.; Fang, Y.; Zhang, B. Org. Bimol. Chem. 2019, 17, 4208.

(g) Sun, K.; Wang, S.; Feng, R.; Zhang, Y.; Wang, X.; Zhang, Z.; Zhang, B. Org. Lett. 2019, 21, 2052.

(h) Sun, K.; Shi, Z.; Liu, Z.; Luan, B.; Zhu, J.; Xue, Y. Org. Lett. 2018, 20, 6687.

[11] (a) Lan, X.; Wang, N.; Xing, Y. Eur. J. Org. Chem. 2017, 5821.

(b) Bag, R.; De, P. B.; Pradhan, S.; Punniyamurthy, T. Eur. J. Org. Chem. 2017, 5424

(c) Romero, R. M.; Wçste, T. H.; MuÇiz, K. Chem. Asian J. 2014, 9, 972.

(d) Chemler, S. R.; Bovino, M. T. ACS Catal. 2013, 3, 1076.

[12] (a) Smart, B. E. Chem. Rev. 1996, 96, 1555.

(b) Shimizu, M.; Hiyama, T. Angew. Chem., Int. Ed. 2005, 44, 214.

(c) Muller, C. K.; Faeh, C.; Diederich, F. Science 2007, 317, 1881

(d) Purser, S.; Moore, P. R.; Swallow, S.; Gouverneur, V. Chem. Soc. Rev. 2008, 37, 320.

(e) Wang, F.; Wang, D.-H.; Mu, X.; Chen, P.-H.; Liu, G.-S. J. Am Chem. Soc. 2014, 136, 10202.

[13] For recent reviews on trifluoromethylation of alkenes, see: (a) Merino, E.; Nevado, C. Chem. Soc. Rev. 2014, 43, 6598. (b) Egami, H.; Sodeoka, M. Angew. Chem. Int. Ed. 2014, 53, 8294.

[14] For selected examples, see: (a) Li, L.; Gu, Q.; Wang, N.; Song, P.; Li, Z.; Li, X.; Wang, F.; Liu, X. Chem. Commun. 2017, 53, 4038.

(b) Koike, T.; Akita, M. Acc. Chem. Res. 2016, 49, 1937.

(c) Ye, J.; Song, L.; Zhou, W.; Ju, T.; Yin, Z.; Yan, S.; Zhang, Z.; Li, J.; Yu, D. Angew. Chem., Int. Ed. 2016, 55, 10022.

(d) Liu, C.; Lu, Q.; Huang, Z.; Zhang, J.; Liao, F.; Peng, P.; Lei, A. Org. Lett. 2015, 17, 6034.

(e) Yang, B.; Xu, X.; Qing, F. Org. Lett. 2015, 17, 1906.

[15] Recent examples for trifluoromethylative alkene difunctionalization of $N$-arylacrylamides: (a) Kawamura, S.; Sodeoka, M. Angew. Chem., Int. Ed. 2016, 55, 8740.

(b) Guo, J.; Wu, R.; Jin, J.; Tian, S. Org. Lett. 2016, 18, 3850

(c) Liu, C.; Zhao, W.; Huang, Y.; Wang, H.; Zhang, B. Tetrahedron 2015, 71, 4344.

[16] Synthesis of $\mathrm{CF}_{3} \mathrm{CH}_{2}$-containing oxindoles from acryl sulfonamides: (a) Zheng, L.; Yang, C.; Xu, Z.; Gao, F.; Xia, W. J. Org. Chem. 2015, 80, 5730 .

(b) Li, L.; Deng, M.; Zheng, S.; Xiong, Y.; Tan, B.; Liu, X. Org. Lett. 2014, 16, 504

(c) Kong, W.; Casimiro, M.; Merino, E.; Nevado, C. J. Am. Chem. Soc. 2013, 135, 14480.

[17] Bertrand, F.; Pevere, V.; Quiclet-Sire, B.; Zard, S. Z. Org. Lett. 2001, 3, 1069.

[18] Egami, H.; Shimizu, R.; Kawamura, S.; Sodeoka, M. Angew. Chem., Int. Ed. 2013, 52, 4000.

[19] Kawamura, S.; Dosei, K.; Valverde, E.; Ushida, K.; Sodeoka, M. J. Org. Chem. 2017, 82, 12539.

[20] Dai, J.; Fang, C.; Xiao, B.; Yi, J.; Xu, J.; Liu, Z.; Lu, X.; Liu, L.; Fu, Y. J. Am. Chem. Soc. 2013, 135, 8436. 
[21] Zheng, J.; Chen, P.; Yuan, Y.; Cheng, J. J. Org. Chem. 2017, 82, 5790.

[22] Liang, D.; Dong, Q.; Xu, P.; Dong, Y.; Li, W.; Ma, Y. J. Org. Chem. 2018, 83, 11978 .

[23] (a) Yang, B.; Xu, X.; Qing, F. Chin. J. Chem. 2016, 34, 465. (b) Huang, H.; Yan, H.; Gao, G.; Yang, C.; Xia, W. Asian J. Org. Chem. 2015, 4, 674.

(c) Lu, Q.; Liu, C.; Huang, Z.; Ma, Y.; Zhang, J.; Lei, A. Chem. Commun. 2014, 50, 14101.

[24] (a) Wu, T.; Mu, X.; Liu, G. Angew. Chem., Int. Ed. 2011, 50, 12578. (b) Zhang, H.; Chen, P.; Liu, G. Synlett 2012, 23, 2749.

[25] Krys, P.; Matyjaszewski, K. Eur. Polym. J. 2017, 89, 482.

[26] Studer, A.; Curran, D. P. Angew. Chem. Int. Ed. 2016, 55, 58.

[27] Recent examples see: (a) Li, Z.; Yu, H.; Bolm, C. Angew. Chem., Int. Ed. 2017, 56, 9532.

(b) Sato, M.; Azuma, H.; Daigaku, A.; Sato, S.; Takasu, K.; Okano, K.; Tokuyama, H. Angew. Chem., Int. Ed. 2017, 56, 1087.

(c) Ren, S.; Zhang, F.; Qi, J.; Huang, Y.; Xu, A.; Yan, H.; Wang, Y. J. Am. Chem. Soc. 2017, 139, 6050.

(d) Gharpure, S. J.; Shelke, Y. G. Org. Lett. 2017, 19, 5022.

(e) Zhang, H.; Ma, S.; Xing, Z.; Liu, L.; Fang, B.; Xie, X.; She, X. Org. Chem. Front. 2017, 4, 2211.

(f) Liu, X.; Luo, X.; Wu, Z.; Cui, X.; He,Y.; Huang, G. J. Org. Chem. 2017, 82, 2107.

[28] (a) Irudayanathan, F. M.; Lee, S. Org. Lett. 2017, 19, 2318.

(b) Liu, B.; Wang, C.; Hu, M.; Song, R.; Chen, F.; Li, J. Chem. Commun. 2017, 53, 1265.

(c) Song, W.; Yan, P.; Shen, D.; Chen, Z.; Zeng, X.; Zhong, G. J. Org. Chem. 2017, 82, 4444.

(d) Lan, X.; Wang, N.; Bai, C.; Lan, C.; Zhang, T.; Chen, S.; Xing, Y. Org. Lett. 2016, 18, 5986.

(e) Deng, Y.; Tang, S.; Ding, G.; Wang, M.; Li, J.; Li, Z.; Yuan, L.; Sheng, R. Org. Biomol. Chem. 2016, 14, 9348.

(f) Gao, B.; Xie, Y.; Yang, L.; Huang, H. Org. Biomol. Chem. 2016, 14, 2399.

(g) Xie, Y.; Guo, S.; Wu, L.; Xia, C.; Huang, H. Angew. Chem., Int. Ed. 2015, 54, 5900.

(h) Rong, G.; Mao, J.; Zheng, Y.; Yao, R.; Xu, X. Chem. Commun. 2015, 51, 13822.

[29] Li, Y.; Chang, Y.; Li, Y.; Cao,C.; Yang, J.; Wang ,B.; Liang, D. Adv. Synth. Catal. 2018, 360, 2488.

[30] (a) Gribble, G. W. Acc. Chem. Res. 1998, 31, 141.

(b) Vaillancourt, F. H.; Yeh, E.; Vosburg, D. A.; Garneau-Tsodikova,

S.; Walsh, C. T. Chem. Rev. 2006, 106, 3364.

(c) Wang, J.; Sánchez-Roselló, M.; Acena, J. L.; Pozo, C. del; Sorochinsky, A. E.; Fustero, S.; Soloshonok V. A.; Liu, H. Chem. Rev. 2014, 114, 2432.

(d) Paul C.; Pohnert, G. Nat. Prod. Rep. 2011, 28, 186.

(e) Latham, J.; Brandenburger, E.; Shepherd, S. A.; Menon, B. R. K.; Micklefield, J. Chem. Rev. 2018, 118, 232.

[31] (a) Sitachitta, N.; Rossi, J.; Roberts, M. A.; Gerwick, W. H.; Fletcher M. D.; Willis, C. L. J. Am. Chem. Soc. 1998, 120, 7131. (b) Owusu-Ansah, E.; Durow, A. C.; Harding, J. R.; Jordan ,A. C.; O'Connell S.J.; Willis, C. L. Org. Biomol. Chem. 2011, 9, 265.

(c) Yu, H.-Y.; Bao,L.-J.; Liang ,Y.; Zeng, E. Y. Environ. Sci. Technol. 2011, 45, 5245 .

(d) Wagner, C.; Omari M. E.; König, G. M. J. Nat. Prod. 2009, 72, 540 .

(e) Ardá, A.; Soengas, R. G.; Nieto, M. I.; Jiménez, C.; Rodríguez, J. Org. Lett. 2008, 10, 2175.

(f) Orjala, J. O.; Gerwick, W. H. J. Nat. Prod. 1996, 59, 427.

(g) Nguyen,V.-A.; Willis, C. L.; Gerwick, W. H. Chem. Commun. 2001, 1934.

(h) Durow, A. C.; Long, G. C.; O'Connell, S. J.; Willis, C. L. Org. Lett. 2006, 8, 5401.

[32] Pan, C.; Gao, D.; Yang, Z.; Wu, C.; Yu, J. Org. Biomol. Chem. 2018, 16, 5752 .
[33] Simmons, E. M.; Hartwig, J. Angew. Chem., Int. Ed. 2012, 51, 3066 .

[34] (a) Chan, C.-W.; Zhou, Z.; Chan, A. S. C.; Yu, W.-Y. Org. Lett. 2010, 12, 3296.

(b) Jia, X.; Zhang, S.; Wang, W.; Luo, F.; Cheng, J. Org. Lett. 2009, 11,3120 .

(c) Tang, B.-X.; Song, R.-J.; Li, J.-H. J. Am. Chem. Soc. 2010, 132, 8900 .

(d) Wu, Y.; Li, B.; Mao, F.; Li, X.; Kwong, F. Y. Org. Lett. 2011, 13, 3258 .

[35] Pan,C.; Yang, Z.; Gao, D.; Yu, J. Org. Biomol. Chem. 2018, 16, 6035 .

[36] (a) Tercel, M.; Lee, H. H.; Mehta, S. Y.; Youte Tendoung, J.-J.; Bai, S. Y.; Liyanage, H. D. S.; Pruijn, F. B. J. Med. Chem. 2017, 60, 5834 .

(b) Yan, J.; Chen, J.; Zhang, S.; Hu, J.; Huang, L.; Li, X. J. Med. Chem. 2016, 59, 5264

(c) Palmerini, C. A.; Tartacca, F.; Mazzoni, M.; Granieri, L.; Goracci, L.; Scrascia, A.; Lepri, S. Eur. J. Med. Chem. 2015, 102, 403. (d) Na, Z.; Pan, S.; Uttamchandani, M.; Yao, S. Q. Angew. Chem., Int. Ed. 2014, 53, 8421.

(e) Kandekar, S.; Preet, R.; Kashyap, M.; Mu, R. P.; Mohapatra, P.; Das, D.; Satapathy, S. R.; Siddharth, S.; Jain, V.; Choudhuri, M.; Kundu, C. N.; Guchhait, S. K.; Bharatam, P. V. ChemMedChem 2013, 8, 1873.

(f) Zhao, R. Y.; Erickson, H. K.; Leece, B. A.; Reid, E. E.; Goldmacher, V. S.; Lambert, J. M.; Chari, R. V. J. J. Med. Chem. 2012, 55, 766 .

[37] (a) Chansaenpak, K.; Wang, M.; Liu, S.; Wu, Z.; Yuan, H.; Conti, P. S.; Li, Z.; Gabbaï, F. P. RSC Adv. 2016, 6, 23126.

(b) Cai, Z.; Ouyang, Q.; Zeng, D.; Nguyen, K. N.; Modi, J.; Wang, L.; White, A. G.; Rogers, B. E.; Xie, X.; Anderson, C. J. J. Med. Chem. 2014, 57, 6019.

[38] (a) Dousson, C.; Alexandre, F.; Amador, A.; Bonaric, S.; Bot, S.; Caillet, C.; Convard, T.; da Costa, D.; Lioure, M.; Roland, A.; Rosinovsky, E.; Maldonado, S.; Parsy, C.; Trochet, C.; Storer, R.; Stewart, A.; Wang, J.; Mayes, B. A.; Musiu, C.; Poddesu, B.; Vargiu, L.; Liuzzi, M.; Moussa, A.; Jakubik, J.; Hubbard, L.; Seifer, M.; Standring, D. J. Med. Chem. 2016, 59, 1891.

(b) Alexandre, F.; Amador, A.; Bot, S.; Caillet, C.; Convard, T.; Jakubik, J.; Musiu, C.; Poddesu, B.; Vargiu, L.; Liuzzi, M.; Roland, A.; Seifer, M.; Standring, D.; Storer, R.; Dousson, C. B. J. Med. Chem. 2011, 54, 392

[39] Okon, A.; Matos de Souza, M. R.; Shah, R.; Amorim, R.; da Costa, L. J.; Wagner, C. R. ACS Med. Chem. Lett. 2017, 8, 958

[40] Li, M.; Nyantakyi, S. A.; Gopal, P.; Aziz, D. B.; Dick, T.; Go, M. ACS Med. Chem. Lett. 2017, 8, 1165.

[41] (a) Fricke, J.; Blei, F.; Hoffmeister, D. Angew. Chem., Int. Ed. 2017, 56,12352 .

(b) Hofmann, A.; Heim, R.; Brack, A.; Kobel, H.; Frey, A.; Ott, H.; Petrzilka, T.; Troxler, F. Helv. Chim. Acta 1959, 42, 1557.

[42] Wu, S.; Cao, Q.; Wang, X.; Cheng, K.; Cheng, Z. Chem. Commun. 2014, 50, 8919.

[43] Vaillard, S. E.; Postigo, A.; Rossi, R. A. J. Org. Chem. 2002, 67, 8500 .

[44] Gagosz, F.; Zard, S. Z. Synlett 2003, 387.

[45] Bruch, A.; Ambrosius, A.; Fröhlich, R.; Studer, A.; Guthrie, D. B.; Zhang, H.; Curran, D. P. J. Am. Chem. Soc. 2010 , 132, 11452.

[46] Bruch, A.; Fröhlich, R.; Grimme, S.; Studer, A.; Curran, D. P. J. Am. Chem. Soc. 2011, 133, 16270.

[47] Liang, D.; Ge, D.; Lü, Y.; Huang, W.; Wang, B.; Li, W. J. Org. Chem. 2018, 83, 4681.

[48] (a) Xu, J.; Yu, X.; Song, Q. Org. Lett. 2017, 19, 980.

(b) Li, Y.; Sun, M.; Wang, H.; Tian, Q.; Yang, S. Angew. Chem., Int. Ed. 2013, 52, 3972.

(c) Zheng, J.; Zhang, Y.; Wang, D.; Cui, S. Org. Lett. 2016, 18, 1768 .

(Lu, Y.) 